\title{
Analyzing students' reasoning about the inverse-square law through the lens of dual processing theories
}

\author{
Daniel B. Marsh and Rabindra R. Bajracharya \\ Chemical and Physical Sciences Department, Missouri Southern State University, Joplin, MO, 64801
}

We discuss an investigation exploring students' reasoning about the inverse-square law using the perspectives of dual processing. We find that students in both introductory mathematics and physics courses have difficulties with the law when it is presented in multiple representations. More than half of the students who provided incorrect responses to the written surveys inappropriately based their reasoning on various cues in the questions without engaging in genuine conceptual thinking. One common reasoning students provided was based on the inverse relationship rather than the inverse-square relationship between two variables. We suggest that the reason for this could be due to the students' choice of an intuitive and automatic processing (system 1) over a controlled and conscious processing (system 2) while responding to the questions.

2019 PERC Proceedings edited by Cao, Wolf, and Bennett; Peer-reviewed, doi.org/10.1119/perc.2019.pr.Marsh Published by the American Association of Physics Teachers under a Creative Commons Attribution 4.0 license. Further distribution must maintain attribution to the article's authors, cover page, and DOI. 


\section{INTRODUCTION}

The understanding and implementation of physics concepts often involve the application of relevant mathematical knowledge and skills. Students lacking and/or failing to apply relevant mathematical knowledge and skills have shown difficulties with reasoning and/or solving problems in physics contexts [1-3]. For example, Meltzer showed using a physics concept survey that there was a significant correlation between normalized learning gain and students' pre-instruction mathematics skill [1].

One mathematical concept that is obeyed by various physical phenomena is the inverse-square law, such as gravitational, electrostatic, and magnetic forces; intensities of sound and light. Since the law is obeyed by more than one physical phenomena, students' difficulties with the law could adversely affect their understanding of the relevant physics concepts. Thus, an understanding of the mathematical concept underlying the law is important to understand the physical phenomena obeying the law. Although numerous curricular materials have been developed on the law, there has not been any research on teaching and learning of the law in mathematics and physics.

The inverse-square law can be expressed in various representations, such as words, pictures, equations, graphs, and data tables. However, in traditional teaching, much of the emphasis is put on the first three types of representations and not so much on the latter two. A few studies in physics education research have shown a positive correlation between student difficulties with physics concepts and those with the representations used to connect the mathematics and the physics [46]. Particularly, previous studies in other contexts have indicated that students have difficulties with graphical representations. For example, Beichner showed by assessing students' understanding of graphs in kinematics (the Test of Understanding Graphs - Kinematics, or the TUG-K) that students have difficulties interpreting the graphs [7]. Similarly, using tasks from kinematics, McDermott et al. pointed out that students have difficulties connecting graphical representations with physics concepts [8].

To be able to interpret and apply the inverse-square law, students are expected to understand the mathematical concept underlying the law. Students are generally introduced a wide range of algebraic functions in introductory mathematics classes that are usually the pre-requisites for introductory physics classes, such as $y=1 / x, y=1 / x^{2}, y=e^{-x}$. However, several studies in mathematics education have shown that students have difficulties with the concept of function, particularly in graphical representations [9-12]. Our own anecdotal evidence shows that students have difficulties with the graphical representation of the inverse-square law. This led us to impose the research question in this study: "How students respond to and reason about the questions based on the inverse-square law posed in multiple representations?"

\section{THEORETICAL PERSPECTIVES}

Initially, we implemented a Thematic Analysis approach to identify the patterns or themes in students' responses $[13,14]$. The results from the written surveys indicated that students' responses were based not only on their conceptual understanding of the law but also on quick, intuitive, and involuntary processing that is dominated by specific cues in the questions. Our findings were consistent with the findings of some of the previous studies that used the dual processing theories [15-18]. For example, Kahneman used the theories to explain the results of the Cognitive Reflection Test [19] administered at several US universities that manifested common patterns of incorrect responses [20]. According to Kahneman, students have a tendency to invoke automatic and intuitive processing while responding to even simple questions. This inspired us to pursue the dual processing theories for explaining the student reasoning patterns or categories emerging from the Thematic Analysis approach.

According to the dual processing theories $[16,17]$, a large part of the mental processing is attributed to an involuntary mechanism called system 1 . It is the stimulus-driven process and guided only by the salient features of visual scenes. System 1 can be quickly activated by showing the individual a particular location that contains one or more contrasting features. For example, a red circle, in a collection of green circles, may automatically drive one's system 1 processing leading to an increase in attention capture. An individual's attention mechanism that is assumed to be regulated by his or her cognitive machinery, including prior knowledge, nature of tasks, and expectations is called a system 2 . This process is also referred to as a goal-driven mechanism and constitutes deliberately controlled and intentional thinking. In many decision-making contexts, both system 1 and system 2 processes may work simultaneously.

Our results were also consistent with Heckler's perspectives of high- and low-saliency cues [21]. In a survey, Heckler asked students to determine the points at which two cars have same speed on the $x-t$ graphs, one linear and the other curved. About $40 \%$ of the students answered that the cars have same speed where the lines intersect. Even though the cue (the point of intersection) was irrelevant to answering the question, the students still seemed to have focused on the cue. Heckler used the terms 'high- and low-saliency cues' to determine the potential dominant feature. The point of intersection between the two lines is the high-saliency cue. Hecklar also suggested that students' processing is often influenced by the salient features present in a question. According to Heckler, in a competition between high- and low-salience features for visual attention, often the low-salience feature is ignored even when it carries more predictive information. 


\section{METHODOLOGY}

Our purpose was to investigate students' understanding of the inverse-square law in multiple representations. In order to see if their difficulties with the law in physics stem from their difficulties with the corresponding function in mathematics classes, we conducted the study in introductory physics classes and the pre-requisite mathematics classes. The participants were from a small Midwest University. They were recruited on a voluntary basis. The courses considered for this study were representative of the standard introductory courses taught in other US institutions. We chose the classes for this study because the students in the classes had already dealt with the inverse-square function or law in their current and/or previous classes.

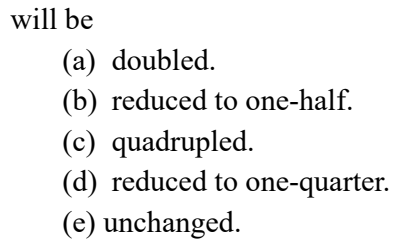

(a) doubled.

(b) reduced to one-half.

(c) quadrupled.

(d) reduced to one-quarter.

(e) unchanged.

Explain your reasoning.

M2. Which of the following expressions represents that $y$ is inversely proportional to square of $x$ ? Explain your reasoning.

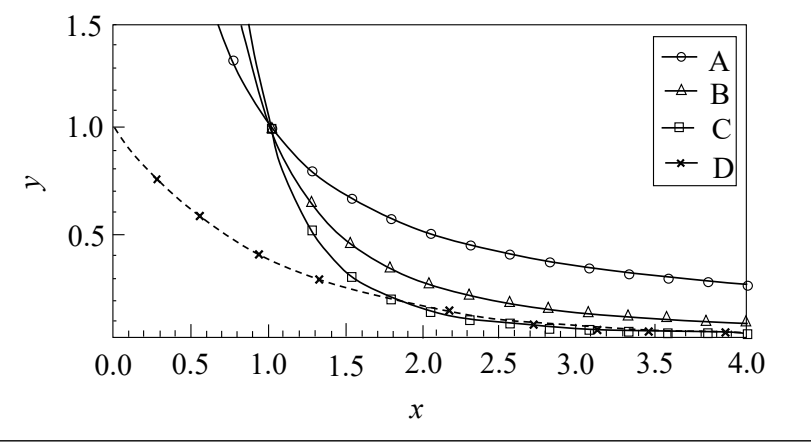

FIG. 1. The survey without any physical context. The survey was administered in mathematics classes.

We constructed two surveys, one without any physics context (version 1) and the other with a physical context (version 2). Each survey consists of four multiple choice questions and prompts for reasoning. The four questions were posed in four different representations, such as words, equations, data tables, and graphs that were based on the inversesquare function/law. Because the types of reasoning provided by the students to the questions with equations and data tables were similar to the reasoning provided to the questions with just words and graphs, we focus only on the questions based on words and graphs in this paper. To choose the correct option and give appropriate reasoning, students are expected to understand how the dependent variable $(y)$ changes with the change in the independent variable $(x)$ if they obey the inverse-square relation. For the graphical question, one
P1. According to the Universal Law of Gravity, every object in the universe attract every other object with a force that is inversely proportional to the square of the distance between the two objects. The gravity force between two masses is $10.0 \mu \mathrm{N}$ when they are $2.0 \mathrm{~cm}$ a part. What would be the gravity force if the distance between them is some how doubled to $4.0 \mathrm{~cm}$ ?
(a) $2.5 \mu \mathrm{N}$.
(b) $5.0 \mu \mathrm{N}$.
(c) $10.0 \mu \mathrm{N}$.
(d) $20.0 \mu \mathrm{N}$
(e) $40.0 \mu \mathrm{N}$

Explain your reasoning.

P2. Which of the graphs below best represents the gravity force between any two objects versus the distance between them? Explain your reasoning.

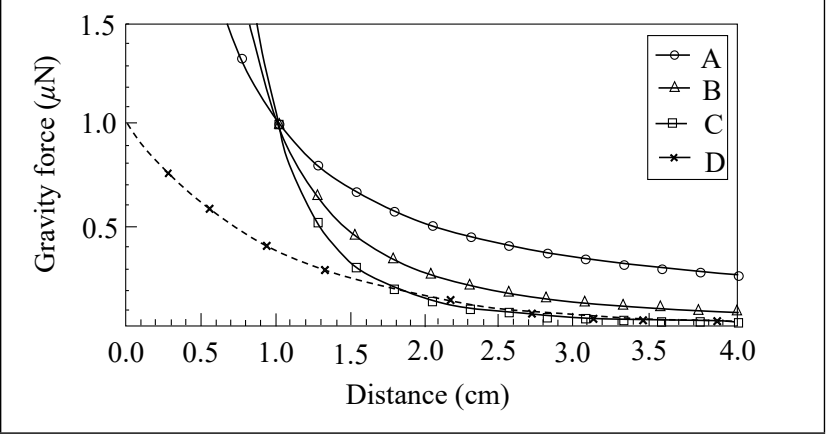

FIG. 2. The survey with a physical context. The survey was administered in physics classes.

can determine the correct curve by inspecting the domain and range values that satisfy the inverse-square relation. For example, the values $(1.00,1.00),(2.00,0.25)$, and $(3.00,0.11)$ on curve B satisfy the desired relation.

Version 1 was administered to two sections of college algebra and a single section of single variable calculus classes. Similarly, version 2 was administered to three sections of second semester algebra-based physics and a single section of first semester calculus-based physics classes.

All of the data were digitized. Individual student responses were later transcribed into spreadsheets for coding and analysis purposes. We analyzed the written data using a Thematic Analysis approach $[13,14]$. Each individual student's reasoning was first identified and compared with that of other students. Then the student reasoning was grouped in a way that included all the important features within the data. This grouping enabled us to produce explanations of the patterns that emerged and the themes that connect them.

\section{RESULT}

Table I depicts the prevalence of students' responses to the survey questions. It shows that the majority of the students chose the incorrect options while responding to the survey questions. We found that the percentage of correct responses 
for both the questions in mathematics and physics classes was less than $40 \%$, except for the question P1 in algebra-based physics II.

TABLE I. Prevalence of student responses to the survey questions. The numbers in bold are percents of correct responses.

\begin{tabular}{|c|c|c|c|c|c|c|}
\hline \multirow{2}{*}{ Class } & \multirow{2}{*}{$\stackrel{\dot{\otimes}}{\mathscr{\theta}}$} & \multicolumn{5}{|c|}{ Percent } \\
\hline & & $\mathrm{A}$ & $\mathrm{B}$ & $\mathrm{C}$ & $\mathrm{D}$ & $\mathrm{E}$ \\
\hline College algebra & M1 & 17 & 59 & 0 & 24 & 0 \\
\hline$(\mathrm{N}=29)$ & M2 & 24 & 14 & 24 & 35 & - \\
\hline Calculus I & M1 & 5 & 48 & 10 & 38 & 0 \\
\hline$(\mathrm{N}=21)$ & M2 & 33 & 19 & 19 & 29 & - \\
\hline Alg.-based phys. II & $\mathrm{P} 1$ & 50 & 30 & 3 & 8 & 8 \\
\hline$(\mathrm{N}=60)$ & $\mathrm{P} 2$ & 17 & 28 & 27 & 22 & - \\
\hline Calc.-based phys. I & $\mathrm{P} 1$ & 38 & 44 & 6 & 6 & 6 \\
\hline$(\mathrm{N}=16)$ & $\mathrm{P} 2$ & 13 & 38 & 25 & 19 & - \\
\hline
\end{tabular}

Students who provided incorrect responses seem to have based their reasoning on the salient cues or features without engaging in genuine conceptual thinking. The results indicate that students may lack a deep conceptual understanding of the inverse-square law. For example, students who based their reasoning on salient graphical features either did not use any mathematical concept, used inappropriate ones, or failed to implement a right one. Those who did not use any mathematical concept wrote "it's the only one that looks different." Similarly, those who used inappropriate ones wrote, " $y$ is dramatically decreasing as $x$ increases" and "at large enough $d$, $F$ goes to 0." Likewise, the students who failed to implement the correct mathematical concept wrote, "it follows the function $y=1 / x^{2}$ " and "the graph represents the inverse of the square of $x . "$ The students selected the wrong curves suggesting that they had difficulty identifying the inverse-square function or law in graphical representation.

Our primary analysis indicated that students used certain cues in the questions that led them to invoke specific math concepts. We found that students' reasoning was based primarily on five different cues in the questions as depicted in Table II. Of the five cues, four were either incomplete, inappropriate, or irrelevant resulting in incorrect answer choices and wrong reasoning. For example, the students who provided the reasoning, "when $x$ is doubled, $y$ is reduced to one half," used the concept of an inversely proportional relationship between $x$ and $y$. Although the question clearly mentioned that $y$ is inversely proportional to the square of $x$, the students apparently failed to notice the word "square" while responding to the question. Similarly, many students knew the effect of distance on gravity superficially, but they did not know the exact inverse square relation between the two.

Table III shows the prevalence of students' reasoning for the options they selected. Because only a small number of student reasoning was based on the "proportional" and the "square" cues, they were merged into a single category called
TABLE II. Student reasoning, the mathematics concepts involved in the reasoning, and the potential cues that invoked the reasoning.

\begin{tabular}{lcc}
\hline \hline Reasoning & Math Concept & Cues \\
\hline As $x \rightarrow 2 x, y \rightarrow y / 4$ & $y \propto 1 / x^{2}$ & Inverse-square \\
As $d \rightarrow 2 d, F \rightarrow F / 4$ & $y \propto 1 / x$ & \\
\hline As $x \rightarrow 2 x, y \rightarrow y / 2$ & $x \& y$ are opposite & Inverse \\
As $d \rightarrow 2 d, F \rightarrow F / 2$ & $y \propto x$ & Proportional \\
\hline As $d \rightarrow 2 d, F \rightarrow 2 F$ & $y \propto x^{2}$ & Square \\
\hline As $d \rightarrow 2 d, F \rightarrow 4 F$ & one or more of & Graphical \\
\hline As $d \uparrow, F \downarrow$ dramatically & above concepts & \\
$F \rightarrow 0$, but never hits 0 &
\end{tabular}

"other." For some classes, the total percentage did not add up to $100 \%$ because some of the students did not provide any reasoning for their choices. The majority of the students who selected the choices incorrectly seemed to be confused between the inverse-square relation and the inversely proportional relation.

TABLE III. Prevalence of student reasoning to the survey questions.

\begin{tabular}{llcccc}
\hline \hline \multirow{2}{*}{ Class } & $\dot{0}$ & \multicolumn{5}{c}{ Percent } \\
\cline { 3 - 7 } & $\stackrel{\Xi}{\tilde{O}}$ & Inverse-sq. & Inverse & Other & Graph. \\
\hline College algebra & M1 & 21 & 55 & 21 & - \\
$(\mathrm{N}=29)$ & $\mathrm{M} 2$ & 10 & 38 & 3 & 24 \\
\hline Calculus I & $\mathrm{M} 1$ & 43 & 43 & 10 & - \\
$(\mathrm{N}=21)$ & $\mathrm{M} 2$ & 29 & 24 & 5 & 19 \\
\hline Alg.-based phys. II & $\mathrm{P} 1$ & 57 & 23 & 8 & - \\
$(\mathrm{N}=60)$ & $\mathrm{P} 2$ & 30 & 22 & 0 & 13 \\
\hline Calc.-based phys. I & $\mathrm{P} 1$ & 25 & 63 & 12 & - \\
$(\mathrm{N}=16)$ & $\mathrm{P} 2$ & 31 & 50 & 0 & 6 \\
\hline \hline
\end{tabular}

The percentage of correct response and reasoning provided by the algebra-based physics students for question P1 was above $50 \%$, which is higher than that provided by the mathematics students for question M1 and the calculus-based physics students for the same question. Chi-squared tests revealed that the difference in the prevalence of student reasoning between the algebra-based physics and the college algebra students was significant, $\chi^{2}(2, N=60)=6.10$ and $\chi^{2}(2, N=81)=13.38$, respectively for the questions with and without a graph at $\alpha<0.05$. The reason for this difference might be due to the fact that the algebra-based physics students had recently dealt with similar questions in the contexts of Coulomb's law and the Biot-Savart law.

Although the percentage of different types of reasoning provided by the mathematics and the physics students was different, as depicted in Table III, the types of incorrect reasoning provided by both groups were mostly similar. This suggests that both the populations may have similar types of 
difficulties with the inverse-square law. For example, for both the populations, the most common incorrect response and reasoning for the questions without a graph are based on the cue "inverse." Figs. 3 and 4 depict how the cue led both the physics and mathematics students to implement an incorrect relationship between the variables resulting in the incorrect responses.

$$
\begin{aligned}
& \text { Neuble the distare, hait the force } \\
& \text { A. Whan distaree is doubled, Force is balved }
\end{aligned}
$$

FIG. 3. A student's reasoning to P1 and P2. The student provided the inversely proportional reasoning to both the questions.

$$
\begin{aligned}
& \text { Inverse is the opposite so if } x \text { doubles, } y \text { is } \\
& \text { reduced to half. } \\
& A \text {, when } x \text { is doubled, } y \text { reduces by } 1 / 2
\end{aligned}
$$

FIG. 4. A student's reasoning to M1 and M2. The student provided the inversely proportional reasoning to both the questions.

The data also show that many students performed worse in the graphical questions than in the word questions, except for the calculus-based introductory class. As discussed earlier, although a few students exhibited the correct mathematical form (i.e., $\left.1 / x^{2}\right)$, they were still unable to identify the correct graph. This corroborates with the previous research on students' interpretation of graphs in other contexts, which indicated that students, in general, have difficulty with graphical representations [9-12].

\section{DISCUSSION AND CONCLUSIONS}

The survey results indicated that many students have several difficulties with the inverse-square law and its corresponding mathematical function presented in multiple representations. The majority of students who provided the correct response used the inverse-square relation or law accurately, indicating that they were engaged in genuine cognitive processes.

Similar to the findings of discussed by Kahneman, we found that the students who responded incorrectly may be using processes that are inadequately cued by the specific words in a question [20]. For example, the students in our study showed a tendency to use an inversely proportional relationship rather than an inverse square relationship. They also exhibited a tendency to use irrelevant and inappropriate salient features while dealing with graphs. A small number of students applied: a directly proportional relation, a square relation, or an inverse of a function while responding to the given questions.

We assert that those who responded correctly followed system 2 processes which invoked the correct relationship between the two variables. In contrast, those who responded incorrectly gave responses that were consistent with system 1 processes. Their answers indicate that they failed to establish the correct relationship between the given variables. Similar to Heckler, we consider that the correct responders identified the relevant cue/s present in the questions, whereas the incorrect responders focused on salient cues or features resulting in incorrect reasoning and incorrect responses [21]. Thus it may be inferred that students' choice of appropriate or inappropriate features is dependent upon whether they base their reasoning on system 1 processes or system 2 processes.

The teaching implication of this study is that more emphasis needs to be put on multiple representations, particularly on graphical representations, while teaching the inversesquare function in mathematics and the inverse-square law in physics. We believe that the use of multiple representations address the difficulties of the students that are not engaged in genuine cognitive processes, but rather in automatic and involuntary processes. Multiple representations also provide students additional ways that lead to the use of higher level cognitive processes.

Instructors should also pay close attention to the features in questions that may invoke incorrect concepts because our study indicates that the wording and representations of the questions may affect how student perceive the questions.

\section{ACKNOWLEDGMENTS}

We thank all the instructors for allowing us to collect data in their classes. We also thank all the students who voluntarily participated in the written surveys.
[1] D. E. Meltzer, The relationship between mathematics preparation and conceptual learning gains in physics: A possible "hidden variable" in diagnostic pretest scores, American Journal of Physics 70, 1259 (2002).

[2] J. M. Buick, Investigating the correlation between mathematical pre-knowledge and learning gains in service physics, European Journal of Physics 28, 1073 (2007).

[3] R. R. Bajracharya and J. R. Thompson, Analytical derivation: An epistemic game for solving mathematically based physics problems, Phys. Rev. Phys. Educ. Res. 12, 010124 (2016).

[4] D. E. Meltzer, Relation between students' problem-solving performance and representational format, American Journal of Physics 73, 463 (2005).

[5] P. B. Kohl, Effects of representation on students solving physics problems: A fine-grained characterization, Physical Review Special Topics - Physics Education Research 2, 10.1103/PhysRevSTPER.2.010106 (2006).

[6] P. B. Kohl, Student representational competence and self- 
assessment when solving physics problems, Physical Review Special Topics - Physics Education Research 1, 10.1103/PhysRevSTPER.1.010104 (2005).

[7] R. J. Beichner, Testing student interpretation of kinematics graphs, American Journal of Physics 62, 750 (1994).

[8] L. C. McDermott, M. L. Rosenquist, and E. H. Van Zee, Student difficulties in connecting graphs and physics: Examples from kinematics, American Journal of Physics 55, 503 (1987).

[9] D. Tall and M. Bakar, Students' mental prototypes for functions and graphs, International Journal of Mathematical Education in Science and Technology 23, 39 (1992).

[10] S. Vinner, The function concept as a prototype for problems in mathematics learning, in The concept of function: Aspects of epistemology and pedagogy, Vol. 25, edited by G. H. . E. Dubinsky (MAA Notes, 1992) pp. 195-213.

[11] S. Vinner and T. Dreyfus, Images and definitions for the concept of function, Journal for Research in Mathematics Education 20, 356 (1989).

[12] T. Eisenberg and T. Dreyfus, On visual versus analytical thinking in mathematics, in Proceedings of the Tenth International Conference for Psychology of Mathematics Education (1986) pp. 153-158.

[13] J. Aronson, A pragmatic view of thematic analysis, The qualitative report 2, 1 (1995).
[14] V. Braun and V. Clarke, Using thematic analysis in psychology, Qualitative research in psychology 3, 77 (2006).

[15] J. S. B. T. Evans, The heuristic-analytic theory of reasoning: Extension and evaluation, Psychonomic Bulletin Review 13, 378 (2006).

[16] D. Kahneman, Thinking, Fast and Slow (Macmillan, New York, 2011).

[17] C. R. Gette, Probing student reasoning approaches through the lens of dual-process theories: A case study in buoyancy, Physical Review Physics Education Research 14, 10.1103/PhysRevPhysEducRes.14.010113 (2018).

[18] A. K. Wood, Can dual processing theory explain physics students' performance on the force concept inventory?, Physical Review Physics Education Research 12, 10.1103/PhysRevPhysEducRes.12.023101 (2016).

[19] S. Frederick, Cognitive reflection and decision making, Journal of Economic Perspectives 19, 25 (2005).

[20] D. Kahneman, A perspective on judgment and choice: Mapping bounded rationality, American Psychologist 58, 697 (2003).

[21] A. F. Heckler, The ubiquitous patterns of incorrect answers to science questions: The role of automatic, bottom-up processes, Psychology of Learning and Motivation-Advances in Research and Theory 55, 227 (2011). 